\title{
Retroviral gene therapy with an immunoglobulin-antigen fusion construct protects from experimental autoimmune uveitis
}

\author{
Rajeev K. Agarwal, ${ }^{1}$ Yubin Kang, ${ }^{2}$ Elias Zambidis, ${ }^{2}$ David W. Scott, ${ }^{2}$ \\ Chi-Chao Chan, ${ }^{1}$ and Rachel R. Caspi ${ }^{1}$ \\ ${ }^{1}$ Laboratory of Immunology, National Eye Institute, NIH, Bethesda, Maryland, USA \\ ${ }^{2}$ Department of Immunology, Holland Laboratory of the American Red Cross, Rockville, Maryland, USA \\ Address correspondence to: Rachel R. Caspi, Laboratory of Immunology, National Eye Institute, 10 Center Drive MSC 1857, \\ 10/10N222, Bethesda, Maryland 20892-1857, USA. Phone: (301) 435-4555; Fax: (301) 480-6668; E-mail: rcaspi@helix.nih.gov.
}

Received for publication December 15, 1999, and accepted in revised form May 31, 2000.

Immunoglobulins can serve as tolerogenic carriers for antigens, and B cells can function as tolerogenic antigen-presenting cells. We used this principle to design a strategy for gene therapy of experimental autoimmune uveitis, a cell-mediated autoimmune disease model for human uveitis induced with the uveitogenic interphotoreceptor retinoid-binding protein (IRBP). A retroviral vector was constructed containing a major uveitogenic IRBP epitope in frame with mouse IgG1 heavy chain. This construct was used to transduce peripheral B cells, which were infused into syngeneic recipients. A single infusion of transduced cells, 10 days before uveitogenic challenge, protected mice from clinical disease induced with the epitope or with the native IRBP protein. Protected mice had reduced antigen-specific responses, but showed no evidence for a classic Th1/Th2 response shift or for generalized anergy. Protection was not transferable, arguing against a mechanism dependent on regulatory cells. Importantly, the treatment was protective when initiated 7 days after uveitogenic immunization or concurrently with adoptive transfer of primed uveitogenic $T$ cells. We suggest that this form of gene therapy can induce epitope-specific protection not only in naive, but also in already primed recipients, thus providing a protocol for treatment of established autoimmunity.

J. Clin. Invest. 106:245-252 (2000).

\section{Introduction}

The failure to discriminate between self and nonself leads to clinical manifestations of autoimmunity. A number of experimental procedures have been proposed to induce protective tolerance to autoantigens (1-5); however, tolerogenesis in an already immune host has been difficult to achieve. Based on the tolerogenic properties of immunoglobulin carriers combined with the efficacy of B-cell antigen presentation for unresponsiveness, we demonstrated previously that a retroviral vector encoding an immunodominant peptide of phage $\lambda$ repressor protein in frame with a murine IgG1 heavy chain was tolerogenic when transduced into bone marrow cells or LPS-stimulated B cells (6). Genetically compatible recipients of the transduced cells were rendered hyporesponsive to the $\lambda$ repressor epitope.

In the present study, we have built on this model antigen system as the basis of an approach for induction of protective tolerance from autoimmune disease. We used the model of experimental autoimmune uveitis (EAU), a T-cell mediated disease that targets the neural retina. EAU can be induced in susceptible animals by immunization with retinal antigens or their fragments or by adoptive transfer of T cells specific to these antigens $(7,8)$. The underlying immunopathogenic mechanisms are shared by other cell-mediated autoimmune diseases, permitting a generalization of therapeutic approaches and conclusions developed in the uveitis model to other systems. Importantly, EAU serves as a model of human autoimmune uveitis, which is estimated to cause $10 \%$ of the cases of severe visual impairment. Current treatments for uveitis employ systemic medications that have severe side effects and are globally immunosuppressive (9). Thus, there is an urgent need to develop effective immunotherapeutic strategies that are nontoxic and that specifically target the pathogenic cell population.

To test whether tolerance induction by gene transfer could be used to ameliorate autoimmunity, we engineered a chimeric retrovirus encoding a major pathogenic epitope (residues 161-180 of mouse interphotoreceptor retinoid-binding protein [IRBP]) (10) in frame with mouse IgG1 heavy chain. Recipients of B cells transduced with the chimeric retrovirus and challenged with a uveitogenic regimen of the 161-180 epitope were significantly protected from disease. Most importantly, this gene therapy approach was effective even when initiated 7 days after uveitogenic immunization, when uveitogenic effectors are already primed, although a more intense tolerogenic regimen was required. We suggest that this form of gene therapy can be used to induce epitope-specific protection not only 
in naive but also in already primed recipients pointing to a possible clinical applicability of this approach.

\section{Methods}

Animals. Female B10.RIII (H-2 ${ }^{\mathrm{r}}$ ) mice, 6-8 weeks old, were purchased from the Jackson Laboratories (Bar Harbor, Maine, USA) and were housed under pathogen-free conditions. Animal care and use was in compliance with institutional guidelines.

Synthetic peptide. The murine 161-180 peptide (SGIPYVISYLHPGNTVMHVD) and its human homologue (SGIPYIISYLHPGNTILHVD) were synthesized on a PE Applied Biosystems (Foster City, California, USA) peptide synthesizer as described previously (10).

Retroviral constructs and virus producer cell lines. The MBAE retroviral vector encoding the 12-26 epitope of bacteriophage $\lambda \mathrm{cI}$ repressor protein fused in frame to mouse IgG1 heavy chain and its viral producer cell line (F6P), described previously (6), were used as a mock control in the current study. The IRBP161-180-IgGMBAE retroviral vector, as well as the high-titer $\left(\sim 10^{6}\right.$ neomycin-resistant NIH 3T3 CFU per milliliter) and helper virus-free packaging cell line (\#52/139), were similarly generated (ref. 6; Figure 1). Viral producer cell lines were stored in liquid nitrogen and freshly thawed for individual experiments.

Gene transfer into LPS-stimulated B cells for prevention and treatment of EAU. Retroviral-mediated gene transfer into LPS-stimulated (Escherichia coli 055:B5; Sigma Chemical Co., St. Louis, Missouri, USA) B cells has been described $(6,11)$. Briefly, the cells to be transduced (B-cell blasts stimulated with LPS for 24 hours) were cocultured ( $4 \times$ $10^{6}$ cells $/ \mathrm{mL} ; 6-\mathrm{mL}$ cultures) with irradiated $(20 \mathrm{~Gy})$ $52 / 139$ or F6P (mock) virus-producer monolayers in the presence of $6 \mu \mathrm{g} / \mathrm{mL}$ polybrene and $50 \mu \mathrm{g} / \mathrm{mL}$ LPS for 24 hours. The transduced B-cell blasts were injected (30-40 $\times 10^{6}$ cells/mouse, intraperitoneally) into syngeneic recipients. On the basis of experiments with a green fluorescent protein-containing vector, the percentage of productively transduced cells is estimated to be about $10-20 \%$ of the infused inoculum. In a disease-prevention protocol, 8-10 days after receiving the transduced B-cell blasts, the recipient mice were immunized for EAU induction with either the mouse or the human homologue of peptide 161-180 (see below). In a disease-reversal protocol, recipients were given three infusions of transduced B cells on days 7, 9, and 11 after the uveitogenic immunization.

The murine IgG1 heavy chain used in the retroviral vector binds the hapten NIP after assembling with $\lambda$ light chain $(6,11)$. Therefore, production of retroviral constructs can be confirmed by infection of J558L myeloma cell line and measurement of NIP-specific IgG1 in the supernatants. ELISA results showed that J558L cells transduced with m161-180-IgG1 and cI 1226-IgG produced $52 \mathrm{ng} / \mathrm{mL}$ and $68 \mathrm{ng} / \mathrm{mL}$ chimeric $\mathrm{IgG}$, respectively. Gene expression in vivo was verified by the detection of the NIP-specific IgG1 heavy chain in sera of recipient mice $(6,11)$.
EAU induction and scoring. Mice were immunized subcutaneously with murine peptide $(100 \mu \mathrm{g})$ or human peptide $(10-25 \mu \mathrm{g})$ in CFA $(1: 1, \mathrm{vol} / \mathrm{vol})$ supplemented with Mycobacterium tuberculosis to $2.5 \mathrm{mg} / \mathrm{mL}$. Bordetella pertussis toxin (Sigma Chemical Co.) (PTX; $1.0 \mu \mathrm{g}$ in 100 $\mu \mathrm{L})$ was given intraperitoneally with the murine homologue only. EAU by adoptive transfer was induced with $20-40 \times 10^{6}$ pooled lymph node and spleen cells and cultured for 3 days with the human 161-180 peptide, as described (12). Eyes collected for histopathology 21 or 28 days after immunization (10-12 days after adoptive transfer) were fixed and embedded in methacrylate (13). The incidence and severity of EAU on hematoxylin and eosin-stained sections were scored on an arbitrary scale of 0 to 4 , according to a semiquantitative system described earlier $(8,13)$.

The choice of peptide (human or murine) used for challenge depended on the nature of the experiment. For measuring immunological responses the murine peptide is preferred because of the possibility that the human peptide might elicit clones recognizing heterologous specificities absent from the tolerizing mouse peptide irrelevant to disease, which would confound the readout. For EAU induction the human peptide is usually preferred because it obviates the need to use PTX; clones induced to the human peptide that do not recognize autologous specificities would not come into play in a disease readout system.

Immunologic assays. Delayed-type hypersensitivity (DTH) was measured on day 21, 48 hours after challenge with $10 \mu \mathrm{g}$ of the immunizing peptide (or in some cases of IRBP) into the ear pinna (10). The response is calculated as the difference between thickness in micrometers of the antigen-injected and the PBS-injected ears.

Lymphocyte proliferation. Spleens and draining lymph nodes collected on day 21 after immunization were pooled within groups. Triplicate $0.2-\mathrm{mL}$ cultures of $5 \times$ $10^{5}$ cells in DMEM (Hyclone Laboratories Inc., Logan, Utah, USA) supplemented as described $(10,12)$ and containing $1.5 \%$ syngeneic mouse serum were incubated in round-bottomed 96-well plates with graded doses of human or murine $161-180$ peptide or $1 \mu \mathrm{g} / \mathrm{mL}$ of PHA (Murex Biotech Ltd., Dartfield, United Kingdom). The cultures were pulsed after 48 hours with $1 \mu \mathrm{Ci}\left[{ }^{3} \mathrm{H}\right]$ thymidine per well for an additional 18 hours and were harvested and counted by standard liquid scintillation.

Determination of cytokine production. Lymph nodes and spleen cells collected 10 or 21 days after immunization were cultured as for the proliferation assay, except that $10^{6}$ cells $/ 200 \mu \mathrm{L}$ were plated in flat-bottomed 96-well plates with either peptide $(30 \mu \mathrm{M})$ or PHA $(1 \mu \mathrm{g} / \mathrm{mL})$. Supernatants were collected 24 hours later for IL-2 assay and 48 hours later for all other cytokines. IL-2, IL4 , IL-5, and TNF- $\alpha$ were assayed using ELISA kits from Endogen (Woburn, Massachusetts, USA), and IFN- $\gamma$, IL-10, and TGF- $\beta 1$ (total) were assayed using ELISA kits from Genzyme Pharmaceuticals (Cambridge, Massachusetts, USA). 


\section{Figure 1}

Structure of tolerogenic mIRBP161180- $\operatorname{lgG}$ (a) and control cl 12-26IgG (b) constructs. LTR, long terminal repeat; $\Psi$, packaging signal; $\mathrm{Neo}^{\mathrm{R}}$, neomycin resistance gene; P\&E, promoter and enhancer. a

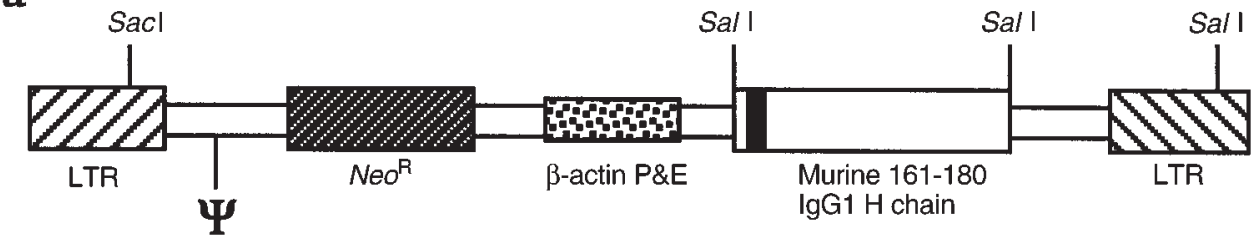

b

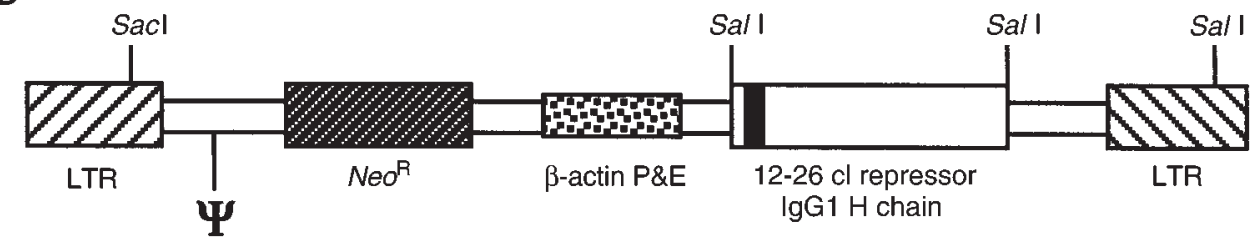

Antibody responses. Peptide-specific IgG2a and IgG1 antibodies were determined in individual sera by ELISA on plates coated with murine or human 161180 peptide $(3 \mu \mathrm{g} / \mathrm{mL})$ and probed with horseradish peroxidase-conjugated goat anti-IgG subclass-specific antibodies (Southern Biotechnology Associates, Birmingham, Alabama, USA), as described earlier for another antigen (14). The concentrations of anti-peptide $\mathrm{Ab}$ isotypes were determined using standard curves generated by coating the wells with anti-isotype antibodies and adding polyclonal isotype Ig standards. Adoptive transfer of B cell-depleted splenocytes. B220-positive cells were depleted from spleen cell suspensions by negative selection with anti-B220-coated magnetic beads, per manufacturer's instructions (Dynal, Lake Success, New York, USA). Flow cytometric analysis (FACScalibur; Becton Dickinson Immunocytometry Systems, San Jose, California, USA) of the depleted population after surface staining with fluoresceinated antibodies for CD19 and CD 3 confirmed less than $0.5 \%$ of residual B cells. Recipient mice were infused intravenously with an equivalent of two donor spleens (approximately $50 \times 10^{6}$ cells) in $0.5 \mathrm{~mL}$ and were challenged with antigen $24-72$ hours after adoptive transfer.

Statistical analysis, reproducibility, and data presentation. Experiments were repeated at least twice; response patterns were highly reproducible. Statistical analysis of EAU scores was by frequency analysis, using Snedecor and Cochran's test for linear trend in proportions (15). Disease severity for each animal was calculated as average of both eyes. Statistical analysis of immunological responses was by 2 -tailed independent $t$ test.

\section{Results}

Recipients of LPS blasts transduced with antigen-IgG construct are protected from EAU. Zambidis et al. (6) reported that infusion of retrovirally transduced cells containing a construct encoding a dominant epitope of phage $\lambda$ repressor protein in frame with an IgG heavy chain (cI 12-26-IgG) led to long-term suppression of the immune response to that epitope. To adapt this methodology to immunotherapy of autoimmune disease, the murine 161-180 epitope of IRBP was engineered into the same vector (m161-180-IgG) (Figure 1).
LPS-stimulated B cells prepared from spleens of naive B10.RIII mice were transduced with $\mathrm{m} 161-180-\mathrm{IgG}$ vector or the control cI 12-26-IgG vector. Recipients of 30-40 million transduced cells were challenged after 8-10 days for induction of EAU by immunization with the murine or the human homologue of peptide 161180 (designated as m161-180 or h161-180, respectively). Note that whereas treatment was always with the autologous murine epitope, uveitogenic challenge was either with the murine or with the human homologue (for reasons stated in Methods). Histopathology of eyes obtained 21 days after immunization, when disease in controls is at its peak, showed that the single infusion of transduced B cells afforded highly significant protection from disease, whether induced by challenge with the autologous murine or the homologous human epitope (Figure 2a). Many of the treated mice remained completely free of disease. Importantly, mice immunized with the native IRBP molecule were also significantly protected, indicating that tolerance to

\section{a}

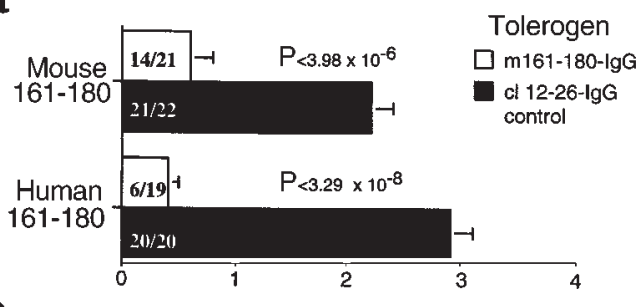

b

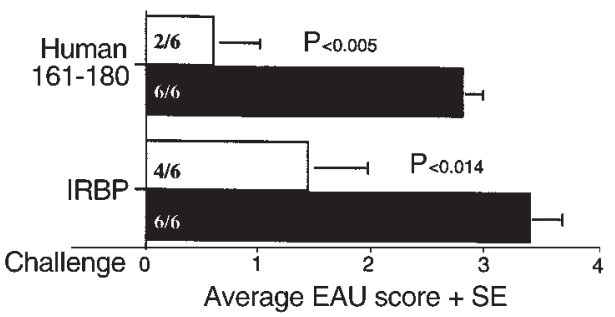

Figure 2

EAU scores in recipients of LPS blasts transduced with mIRBP161180-IgG, infused 8-10 days before uveitogenic challenge with (a) murine 161-180 or human 161-180, or (b) human peptide or whole IRBP. The incidence (number of positive out of total mice) is shown within the bars. The data were compiled from five experiments. 
dominant epitope can afford protection against the whole multiepitope protein (Figure $2 \mathrm{~b}$ ). Typical EAU histopathology in mice that received cells transduced with the tolerogenic versus control vectors is shown in Figure 3. In experiments not shown here, animals challenged with peptide as late as 2 months after the tolerogenic infusion were still protected (data not shown).

To find out whether packaging cells carried over with the transduced $\mathrm{B}$ cells might have contributed to the protection, their percentage in the tolerizing inoculum was assessed by staining cytocentrifuged preparations with Giemsa and by performing a differential count of lymphocytes versus fibroblasts. Contamination by packaging cells was found to be $3-5 \%$. Two million
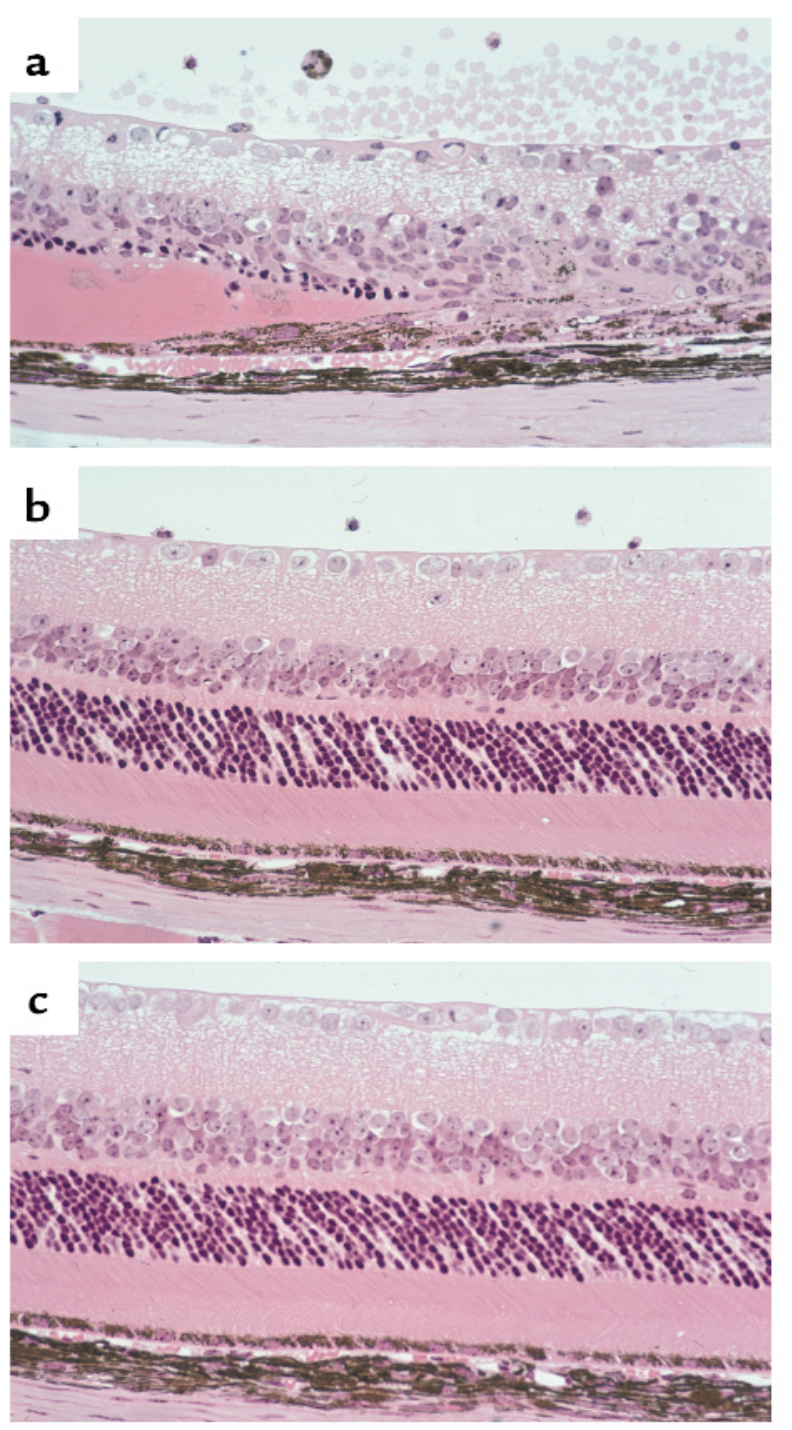

Figure 3

Ocular histology of peptide-immunized mice that had been pretreated with either the LPS-stimulated B cells transduced with the mock control construct (a) or with the tolerogenic construct (b), compared with retina of naive mice (c). Eyes were processed for histology 21 days after uveitogenic immunization. Shown are results after immunization with human peptide. Ocular pathology of mice challenged with the murine construct was essentially identical.
p161-180-IgG retrovirus-producing irradiated packaging cells (equivalent to $6 \%$ contamination) infused into recipient mice had no protective effect whatsoever against peptide challenge, confirming that tolerogenesis was caused by the transduced B-cell blasts.

Cell-mediated and humoral responses in treated mice. Mice infused with transduced LPS blasts as above were challenged for DTH with the immunizing peptide on day 19. Mice that were functionally protected from EAU (whose disease scores are shown in Figure 2) had moderately, albeit significantly, reduced DTH responses $(P$ $<0.002$, data not shown). Draining lymph node and spleen cells collected from these mice on day 21 were tested for proliferation against the immunizing peptides (Figure 4). Mice challenged with the murine peptide had significantly and reproducibly depressed proliferative responses and exhibited a dose-response shift over approximately 1.5 logs of antigen concentration. The effect on in vitro proliferation to human peptide was less pronounced, which probably reflects the fact that the tolerizing peptide and the immunizing peptide are not identical (data not shown).

Antigen-specific cytokine responses were examined 10 and 21 days after immunization. We chose to study responses only of mice immunized with the murine 161-180 homologue so that any cross-reactive responses would not obscure specific hyporesponsiveness. Type 1 /proinflammatory cytokines IL-2, IFN- $\gamma$, and TNF- $\alpha$ and type 2 /anti-inflammatory cytokines IL- 4 , IL-5, IL-10, and TGF $\beta 1$ were assayed by ELISA in 48hour supernatants of cells stimulated with the immunizing peptide. Diminished cytokine responses resembling the effects on DTH and lymphocyte proliferation were seen for IL-2, IFN- $\gamma$, and IL-10 (Figure 5). TNF- $\alpha$ secretion by spleen and lymph node cells of the protected group was half that of the control group on day 10 (but not on day 21), and several other cytokines, including TGF- $\beta$, did not exhibit consistent differences (data not shown).

Antigen-specific IgG1 and IgG2a antibody responses were assayed in individual sera collected from recipients of protective or mock control cells 21 days after a uveitogenic challenge with mouse 161-180 peptide. Because the switch factor for IgG1 is IL-4 and the switch factor for IgG2a is IFN- $\gamma$, these antibody isotypes can also serve as a readout of the Th1 or Th2 bias of the antigen-specific response. Thus, skewing of the IgG1/IgG2a ratio would be indicative of an immune deviation, whereas an overall reduction in both isotypes would indicate a mechanism unrelated to the Th1/Th2 balance. Isotype-specific ELISA showed that, on average, both IgG1 and IgG2a anti-IRBP antibody titers in protected mice were reduced to half the values of controls, although individual titers were variable (Figure 6). Recipients of 161-180-IgG-transduced cells showed a trend toward higher IgG1/IgG2a ratios than the control group. Because of the large individual variability, however, the difference between the group averages did not attain statistical significance ( $t$ test). 


\section{Figure 4}

Lymphocyte proliferation to murine 161-180 epitope in protected mice. Shown is an average of two identical experiments. Counts were normalized to mock control at $30 \mu \mathrm{M}$ peptide after background subtraction (100\%) to compensate for interexperiment variation. (Actual $100 \%$ values for spleen and lymph node, respectively, were 31,650 and 42,900 cpm, with background of 7,000 cpm). The EAU scores of these mice are shown in Figure 2.

Protection from EAU is not transferable. We next wished to examine whether spleens of mice that received the protective treatment contain regulatory cells that could adoptively transfer protection to untreated recipients. Donor mice were given LPS-stimulated B cells transduced with the protective or control retroviral construct. After 10 days, when the animals would normally be challenged for EAU induction, their spleens were removed and depleted of $\mathrm{B} 220^{+}$cells by immunomagnetic beads (resulting in $<0.5 \%$ residual $\mathrm{B}$ cells) to minimize carryover of transduced B cells that could be tolerogenic APCs in the adoptive transfer recipient. Recipients infused with an equivalent of two B cell-depleted donor spleens were challenged for EAU development. Recipients of splenocytes from donors who received the protective treatment developed EAU scores equivalent to scores developed by recipients of control splenocytes, suggesting absence of regulatory cells in the transferred population (Figure 7a). Because this interpretation is based on a negative result, a positive control was used to show that a measurable immune function could be successfully transferred under these conditions. In a parallel experiment, an equivalent number of B cell-depleted splenocytes were able to transfer a DTH response from IRBP-immunized donors to naive recipients (Figure $7 \mathrm{~b}$ ).

Induction of protection in primed recipients. For an immunotherapy strategy to be clinically relevant, it must be effective in an already primed subject. To test whether this kind of retroviral gene therapy would be able to ameliorate disease if administered after priming, we infused LPS blasts transduced with the protective or the control constructs into recipients that had been immunized with human 161-180 seven days earlier.

Clinical onset of EAU in this model normally occurs between 9 to 12 days. To confirm that on day 7 the animals have primed cells that are functional in terms of disease, we extracted spleen and lymph node cells from animals immunized with human 161-180 seven days before, and subjected them to a standard 3-day in vitro activation before adoptive transfer into naive recipients. Recipients of $40 \times 10^{6}$ cells developed EAU with a mean score of 3 , which is directly comparable to scores seen routinely with recipients of an equivalent number of cells extracted 2-3 weeks after immunization.

A single infusion of gene-transduced cells, which was highly effective in protecting preimmune animals, was ineffective in ameliorating disease in primed recipients.

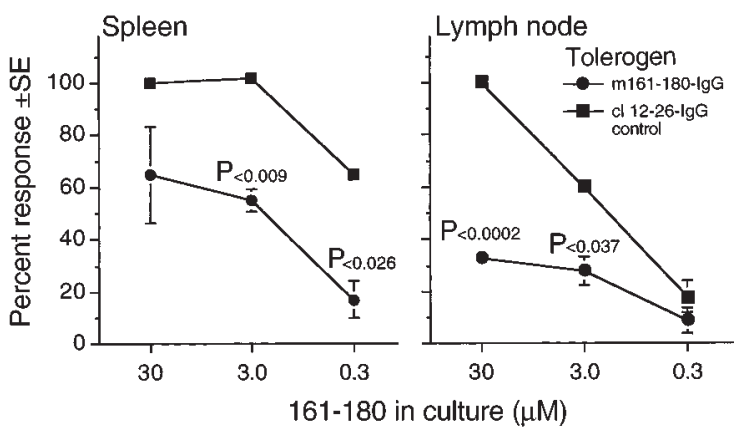

However, three consecutive infusions, given every other day, were highly effective in reducing EAU scores (Figure 8). Furthermore, a single infusion was able to reduce EAU elicited by adoptive transfer of primed $T$ cells. Thus, six of six mice given $30 \times 10^{6} \mathrm{~m} 161-180-\mathrm{IgG}-$ transduced $\mathrm{B}$ cells and challenged 12 hours later by adoptive transfer of uveitogenic T cells from donors immunized with peptide 161-180 were completely protected. In contrast, three of six recipients of mock control T cells and three of five naive recipients developed EAU. This treatment strategy can, therefore, be protective in a situation where primed effector cells have already been generated.

\section{Discussion}

In this study we have used a retroviral gene therapy strategy to prevent or reverse EAU. We demonstrate that recipients of LPS blasts transduced with a tolerogenic construct encoding the murine 161-180 peptide of IRBP in frame with murine IgG1 were highly protected from EAU induced with either the human or the murine uveitogenic peptide homologues and were sig-

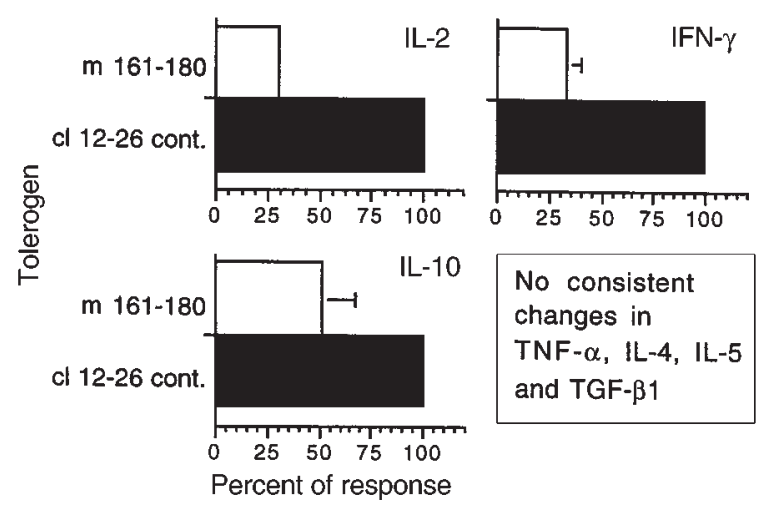

Figure 5

IL-2, IFN- $\gamma$, and IL-10 production by spleen cells of tolerized mice to $30 \mu \mathrm{M}$ peptide. Shown are IFN- $\gamma$ and IL-10 production as assayed 21 days after immunization (average of three experiments) and IL-2 as assayed only 10 days after immunization (single experiment). Values are normalized against the mock control (100\%). Lower level of detectability in picograms per milliliter was 26 for IL-2, 30 for IFN- $\gamma$, and 10 for IL-10. Actual $100 \%$ values in picograms per milliliter were 3,600 for IL-2, 910 for IFN- $\gamma$, and 56 for IL-10. The pattern of IFN- $\gamma$ and IL-10 responses on day 10 was the same as on day 21 , though the absolute amounts secreted were higher. Lymph node cytokine responses were essentially identical to spleen responses. 


\begin{tabular}{cr}
\multicolumn{1}{c}{ Tolerogen } & \multicolumn{1}{c}{$\operatorname{lgG} 1 / \mathrm{lgG} 2 \mathrm{a}$} \\
\hline$\square$ m161-180-lgG & $10.6 \pm 2.1$ \\
cl12-26-lgG & $3.6 \pm 0.5$
\end{tabular}

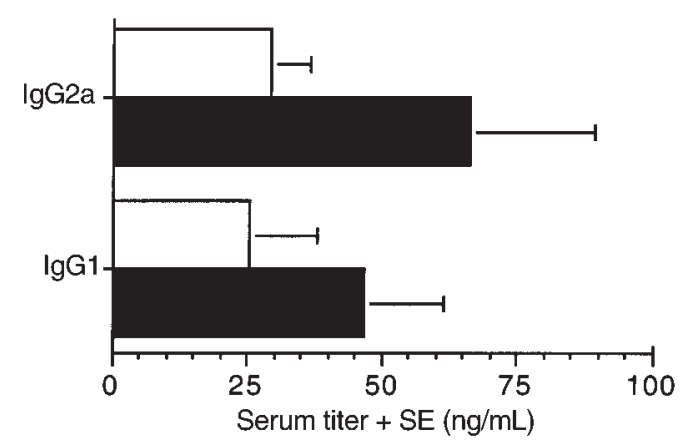

Figure 6

Humoral response to m161-180 in tolerized mice. The bars show average $\lg \mathrm{G} 2 \mathrm{a}$ and $\lg \mathrm{G} 1$ titers of 20 mice compiled from four repeat experiments. The $\operatorname{lgG} 1 / \lg \mathrm{g} 2 \mathrm{a}$ ratio shown at the top was calculated as an average of individual Ig isotype ratios.

nificantly protected from challenge with the whole, multiepitope, IRBP molecule. In experiments not shown here, bone marrow cells transduced with this construct and infused into irradiated recipients had similar protective effects (R.K. Agarwal et al., unpublished observations). It should be pointed out that the term "tolerogenic" is used here to convey the ability to protect from clinical disease, not to indicate lack of an immune response or to imply a particular mechanism.

Whereas the present approach builds on data that demonstrated tolerance induction in a model antigen system of an immunodominant phage $\lambda$ repressor epitope, it was not a given that such a strategy could be adapted to a self antigen in a therapeutic setting. The cI 12-26 epitope is derived from a nonself antigen, to which the T-cell repertoire is of high affinity. In the case of self epitopes, which have escaped the checkpoint of negative selection in the thymus, the repertoire is of medium and low affinity. There is abundant evidence in the literature that strength of signal determines the quality of the Tcell response to an antigenic stimulus to the point that responses to partial agonists can be totally opposite from responses to full agonists. Our study is therefore an important advance that translates findings obtained in a model antigen system to a therapeutic setting.

\section{Figure 7}

EAU (a) and DTH (b) scores in recipients of B cell-depleted splenocytes. (a) EAU scores in recipients of cells from donors who received infusion of LPS blasts transduced with the protective or mock control retroviral constructs. Mice were challenged with the human peptide 24 hours after the infusion and scored for EAU on day 21. (b) DTH scores to IRBP in recipients of spleen cells prepared by the same method from IRBP-immunized or from naive donors (positive control). Recipients were challenged with $10 \mu \mathrm{g}$ of IRBP into the ear pinna 72 hours after transfer. DTH scores were read after 48 hours.

It is important to emphasize that the present approach is not restricted to ocular autoimmunity. There are many tissue-specific autoimmune diseases that share essential mechanisms with uveitis (e.g., experimental autoimmune encephalomyelitis [EAE], diabetes, arthritis, and thyroiditis). The therapeutic approach we describe, modified to incorporate the appropriate antigens, is equally applicable to those other disease entities. Recent data indicate that similar constructs containing myelin basic protein (MBP) are able to protect mice from EAE (M. Melo and D.W. Scott, manuscript in preparation). This enhances the therapeutic value of this strategy in cases where a dominant self epitope is known. Thus, the present approach presents a platform that can be generalized for gene therapy of cell-mediated and, potentially, also of antibody-mediated autoimmune diseases.

In a clinical setting, the patient presents in an immunologically primed state, and continuous recruitment of new pathogenic clones is thought to occur in chronic disease. Importantly, the present regimen afforded protection to both unprimed and primed hosts, although the latter may require repeated tolerogenic infusions. In the present experiments we did not address the question of how long the hyporesponsive state persists beyond 2 months; however, in the cI 12-26 model antigen system tolerance lasts at least 4-8 months (Y. Kang, M. El-Amine, and D.W. Scott, manuscript in preparation). We therefore believe that this system may offer a powerful approach in prevention or amelioration of autoimmune disease in a clinical setting.

Previous studies have used intravenous infusions of antigens chemically coupled to autologous immunoglobulins or cells to induce tolerance $(1,3,16)$. The advantage of the present approach is that it introduces a self-renewing source of tolerogen that establishes residence in the body and essentially becomes part of "self," as opposed to remaining an exogenous treatment whose effects may be transient. Moreover, in already immune individuals the present approach has an additional advantage over an intravenous bolus of an antigen-Ig conjugate in that it avoids introducing a large amount of antigen into the bloodstream, which might trigger an anaphylactic reaction.

There is also a clear advantage to using ex vivo-transduced autologous cells over direct administration of the chimeric retrovirus, because it largely circumvents the

a

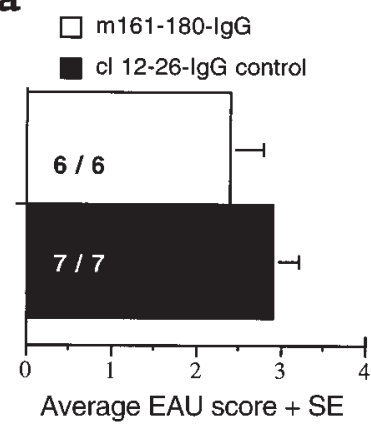

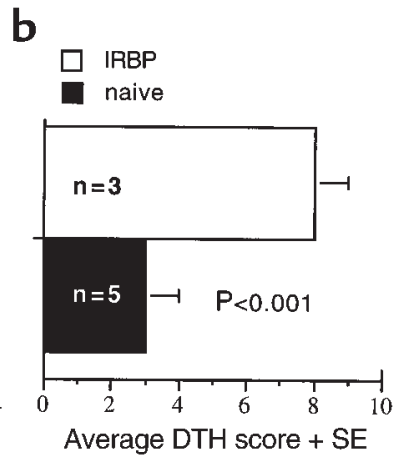


Figure 8

EAU scores in primed recipients of LPS blasts, infused 7 days after uveitogenic challenge with h161-180. The incidence (number of positive out of total mice) is shown within the bars.
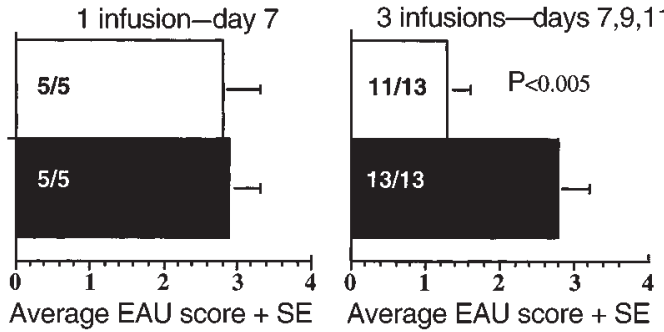

Tolerogen

$\square$ m161-180-lgG

cl 12-26-IgG

control problems inherent in administering immunogenic viral vectors in vivo. This may be particularly important in view of the apparent need for repeated tolerogenic treatments in the primed host. It should be noted that protection was obtained in this system with both LPS blasts and with bone marrow cells (R.K. Agarwal et al., unpublished results) as tolerogenic vehicles. Recently McPherson et al. reported protection from EAU in the rat model using reconstitution of irradiated recipients with bone marrow cells transduced with the retinal soluble antigen (17). Because LPS blasts can be used in nonconditioned recipients, they have advantages over bone marrow genetransfer protocols because of their simplicity and because there is no requirement for myeloablation.

In the current study, a single immunodominant uveitogenic peptide was engineered in frame with the IgG scaffold. Whereas this approach provides an important proof of the concept and, at least in the case of IRBP, also affords significant protection against the whole IRBP molecule, in the future full-length proteins or major domains of autoimmunogenic proteins will need to be employed. In human uveitis, as in other autoimmune diseases, a number of MHC haplotypes have been connected to susceptibility $(9,18)$ and disease-relevant epitopes recognized by different haplotypes are unknown. Moreover, the now well-recognized phenomenon of epitope spreading (19-21) would require covering of multiple epitopes even within the same haplotype. Data in the phage $\lambda$ repressor model system indicates that the full-length protein induces epitope-specific tolerance in diverse $\mathrm{H}-2$ haplotypes (11). Thus, using a full-length autoantigen would have the advantage of allowing different genotypes to process and present the epitopes appropriate for their MHC polymorphisms.

What is the mechanism of tolerance? There can be at least two possible, nonmutually exclusive mechanisms: anergy as a result of antigen presentation by $B$ cells in the absence of costimulation $(22,23)$ or active suppression by regulatory cytokines (5). Whereas lack of costimulatory signals might explain the protective effect of transduced bone marrow cells, it is difficult to invoke for LPS-stimulated B cells because LPS stimulation upregulates B7.1 and B7.2 expression. We do not know how long the LPS blasts remain activated in vivo, and indeed it is possible that expression of the costimulatory molecules is rapidly lost. Nevertheless, some reports indicate that even activated B-cells can present specific epitopes in a tolerogenic manner $(6,24,25)$, raising the possibility that additional mechanisms might be at play. We hypothesize that initial interaction of specific T lymphocytes with activated B cells presenting the uveitogenic epitope can lead to activation and upregulation of CTLA-4 on the T lymphocytes (26). Upon re-encounter of a B7-expressing activated B cell, CTLA-4 gives an "off" signal to the T lymphocyte (27). This possibility is supported by our recent data in the model antigen system of phage $\lambda$ repressor, which suggested that tolerogenic peptide presentation by donor LPS blasts requires compatible class II MHC molecules for tolerance to occur and that anti-CTLA4 treatment interferes under some circumstances with induction of tolerance (28). Studies are in progress to determine the nature of the tolerogenic APCs in these long-term LPS blast recipients.

Some immunoregulatory regimens are able to inhibit EAU by induction of regulatory cytokines (29). In the present study, this does not appear to be the case. Lack of an obvious Th1/Th2 shift in either cytokine patterns or Ig isotypes and an apparently unchanged TGF- $\beta$ response suggest that immune deviation, as it is currently understood, is not playing an important role in the protection. The protection was not transferable, further supporting the notion that induction of regulatory cells is not a primary mechanism. Interestingly, whereas both IL-10 and IFN- $\gamma$ production to antigen were depressed in treated recipients, there was no global suppression of cytokine synthesis. This, together with the partial inhibition of DTH and lymphocyte proliferation, contrasting with the impressive protection from disease, suggests that antigen-specific clones are not deleted per se by the tolerogenic treatment. Partial unresponsiveness, which could reflect tolerance only of high-affinity clones, is therefore sufficient to achieve clinical success in this system. The interpretation that it is the highaffinity clones that are preferentially tolerized by this regimen is also in line with the marked dose-response shift in the proliferation assay. In this context it is interesting to point out a similarity to IRBP-transgenic mice, which express a portion of IRBP containing the 161-180 epitope extraocularly under control of a class II promoter. These mice, which are highly refractory to induction of EAU, also show a 20-fold dose-response shift in antigen-specific proliferation, rather than complete unresponsiveness (30). A thorough dissection of the relative contributions of various possible mechanisms will be made feasible by the development of transgenic mice expressing uveitogenic T-cell receptors. 
In summary, we have demonstrated that gene therapy with autologous cells transduced with a retroviral construct composed of a uveitogenic epitope fused with an isologous IgG molecule can prevent as well as reverse EAU. The effectiveness of this therapy in preimmune as well as in naive recipients opens the possibility of using this approach in a clinical situation when the patient has a preexisting repertoire of lymphocytes primed to an autologous antigen.

\section{Acknowledgments}

This work was supported in part by United States Public Health Service grants R01 AI35622 and T32 GM07356 (to Y. Kang, E. Zambidis, and D.W. Scott). We are grateful to John Nickerson for providing the p313 plasmid. We thank Shu-Hui Sun and Phyllis Silver for valuable help during different parts of the study.

1. Billingham, R.E., Brent, L., and Medawar, P.B. 1953. Actively acquired tolerance of foreign cells. Nature (London). 172:603-606.

2. Chiller, J.M., Habicht, G.S., and Weigle, W.O. 1970. Cellular sites of immunologic unresponsiveness. Proc. Natl. Acad. Sci. USA. 65:551-556.

3. Borel, Y., Lewis, R.M., and Stollar, B.D. 1973. Prevention of murine lupus nephritis by carrier-dependent induction of immunologic tolerance to denatured DNA. Science. 182:76-78.

4. Min, B., Legge, K.L., Pack, C., and Zaghouani, H. 1998. Neonatal exposure to a self-peptide-immunoglobulin chimera circumvents the use of adjuvant and confers resistance to autoimmune disease by a novel mechanism involving interleukin 4 lymph node deviation and interferon gamma-mediated splenic anergy. J. Exp. Med. 188:2007-2017.

5. Garcia, G., and Weiner, H.L. 1999. Manipulation of Th responses by oral tolerance. Curr. Top. Microbiol. Immunol. 238:123-145.

6. Zambidis, E.T., Kurup, A., and Scott, D.W. 1997. Genetically transferred central and peripheral immune tolerance via retroviral-mediated expression of immunogenic epitopes in hematopoietic progenitors or peripheral B lymphocytes. Mol. Med. 3:212-224.

7. Gery, I., Mochizuki, M., and Nussenblatt, R.B. 1986. Retinal specific antigens and immunopathogenic processes they provoke. Prog. Retin. Eye Res. 5:75-109.

8. Caspi, R. 1997. Experimental autoimmune uveoretinitis (EAU): mouse and rat. Current protocols in immunology. J.E. Coligan, A.M. Kruisbeek, D.H. Margulies, E.M. Shevach, and W. Strober, editors. John Wiley \& Sons. New York, New York, USA. January: Unit 15.16.

9. Nussenblatt, R.B., Whitcup, S.M., and Palestine, A.G. 1996. Uveitis: fundamentals and clinical practice. 2nd edition. Mosby-Year-Book Inc. St. Louis, Missouri, USA. 22-26.

10. Silver, P.B., et al. 1995. Identification of a major pathogenic epitope in the human IRBP molecule recognized by mice of the H-2r haplotype, nvest. Ophthalmol. Vis. Sci. 36:946-954.

11. Kang, Y., et al. 1999. Induction of hyporesponsiveness to intact multideterminant foreign protein via retroviral-mediated gene expression: the IgG scaffold is important for induction and maintenance of immunological hyporesponsiveness. Proc. Natl. Acad. Sci. USA. 96:8609-8614.

12. Tarrant, T.K., Silver, P.B., Chan, C.-C., Wiggert, B., and Caspi, R.R. 1998. Endogenous IL-12 is required for induction and expression of experimental autoimmune uveitis. J. Immunol. 161:122-127.

13. Chan, C.-C., et al. 1990. Pathology of experimental autoimmune uveoretinitis in mice. J. Autoimmun. 3:247-255.

14. Rizzo, L.V., DeKruyff, R.H., Umetsu, D.T., and Caspi, R.R. 1995. Regulation of the interaction between Th1 and Th2 T cell clones to provide help for antibody production in vivo. Eur. J. Immunol. 25:708-716.

15. Snedecor, G.W., and Cochran, W.G. 1967. Statistical methods. 6th edition. Iowa State University Press. Ames, Iowa, USA. 246-247.

16. Borel, Y. 1980. Haptens bound to self IgG induce immunologic tolerance, while when coupled to syngeneic spleen cells they induce immune suppression. Immunol. Rev. 50:71-104.

17. McPherson, S.W., Roberts, J.P., and Gregerson, D.S. 1999. Systemic expression of rat soluble retinal antigen induces resistance to experimental autoimmune uveoretinitis. J. Immunol. 163:4269-4276.

18. Caspi, R.R. 1992. Immunogenetic aspects of clinical and experimental uveitis. Reg. Immunol. 4:321-330.

19. Lehmann, P.V., Sercarz, E.E., Forsthuber, T., Dayan, C.M., and Gammon, G. 1993. Determinant spreading and the dynamics of the autoimmune T-cell repertoire. Immunol. Today. 14:203-208.

20. Vanderlugt, C.J., and Miller, S.D. 1996. Epitope spreading. Curr. Opin. Immunol. 8:831-836.

21. McFarland, H.I., et al. 1999. Determinant spreading associated with demyelination in a nonhuman primate model of multiple sclerosis. $J$. Immunol. 162:2384-2390.

22. Eynon, E.E., and Parker, D.C. 1992. Small B cells as antigen-presenting cells in the induction of tolerance to soluble protein antigens. J. Exp. Med. 175:131-138.

23. Fuchs, E.J., and Matzinger, P. 1992. B cells turn off virgin but not memory T cells. Science. 258:1156-1159.

24. Gilbert, K.M., and Weigle, W.O. 1994. Tolerogenicity of resting and activated B cells. J. Exp. Med. 179:249-258.

25. Zambidis, E.T., and Scott, D.W. 1996. Epitope-specific tolerance induction with an engineered immunoglobulin. Proc. Natl. Acad. Sci. USA. 93:5019-5024.

26. Thompson, C.B., and Allison, J.P. 1997. The emerging role of CTLA-4 as an immune attenuator. Immunity. 7:445-450.

27. Chambers, C.A., and Allison, J.P. 1997. Co-stimulation in T cell responses. Curr. Opin. Immunol. 9:396-404.

28. El-Amine, M.M.M., Kang, Y., and Scott, D.W. 1999. Mechanisms of tolerance induction by an Ig-peptide molecule expressed and secreted by $B$ cells. FASEBJ. 13:A280. (Abstr.)

29. Rizzo, L.V., et al. 1994. Interleukin-2 treatment potentiates induction of oral tolerance in a murine model of autoimmunity. J. Clin. Invest. 94:1668-1672.

30. Xu, H., et al. 2000. Transgenic expression of an immunologically privileged retinal antigen in the periphery enhances self tolerance and abrogates susceptibility to autoimmune uveitis. Eur. J. Immunol. 30:272-278. 